\title{
Retinol-binding protein 4 in transthyretin-associated forms of cardiac amyloidosis: differences in the pathobiologies of mutant (ATTRm) and wild-type (ATTRwt) diseases
}

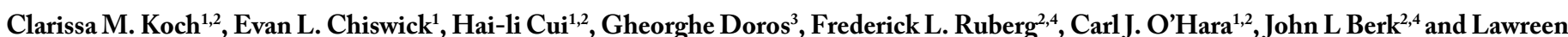
H. Connors ${ }^{1,2 *}$

${ }^{1}$ Department of Pathology and Laboratory Medicine, Boston University School of Medicine, Boston, MA, USA

${ }^{2}$ Amyloidosis Center, Boston University School of Medicine, Boston University School of Medicine, Boston, MA, USA

${ }^{3}$ Department of Biostatistics, Boston University School of Public Health, Boston University School of Medicine, Boston, MA, USA

${ }^{4}$ Department of Medicine, Boston University School of Medicine, Boston, Massachusetts, USA

\begin{abstract}
The role of retinol-binding protein 4 (RBP4), a natural binding partner of plasma circulating transthyretin (TTR), in TTR-associated cardiomyopathies is unknown. RBP4 is a small $(21 \mathrm{kDa})$ protein that normally functions as a transporter for all-trans retinol (Vitamin A) and travels in the bloodstream as a ternary complex bound to TTR. Previous in vitro studies have demonstrated that RBP4 stabilizes native (tetrameric) TTR and prevents its disassembly into monomers, the key step in TTR amyloid fibril formation. Though increased serum concentrations of RBP4 have been reported in non-amyloidotic cardiomyopathies, there is little information about circulating levels in the inherited (ATTRm) and acquired (ATTRwt) forms of TTR-associated cardiac amyloid disease. Our study objectives were to investigate TTR amyloid-infiltrated cardiac tissue for the presence of RBP4 and to compare serum levels of the protein in ATTRm, ATTRwt and controls. We hypothesized that there would be histological and serological differences in RBP4 between ATTR patient and control samples. In the present study, we demonstrate that RBP4 is highly abundant in ATTRm cardiac tissue surrounding amyloid-infiltrated regions and, to a lesser extent, in ATTRwt specimens. Serum levels of RBP4 are significantly lower in ATTRm compared to ATTRwt $(\mathrm{p}<0.0001)$ and healthy controls ( $\mathrm{p}<0.0011)$, with significant correlation between circulating RBP4 concentration and cardiac troponin-I (c-TnI) in our ATTRwt cohort. These data suggest, for the first time, that the pathobiologies of ATTRm and ATTRwt are dissimilar, and provide support for RBP4 as a serum biomarker of amyloid cardiomyopathy in ATTRm.
\end{abstract}

\section{Introduction}

Retinol-binding protein (RBP) is a protein that exists as one of five isoforms. The most abundant and only secreted form is RBP4, a 21 $\mathrm{kDa}$ protein almost exclusively produced by the liver, which transports all-trans retinol (Vitamin A) to target tissues in a ternary complex with transthyretin (TTR). RBP4-TTR complexes are formed in the ER $[1,2]$ and their release from the liver into circulation is dependent on retinol binding to RBP4 [3]. Consequently, $95 \%$ of circulating RBP4 is bound to TTR $[4,5]$. The serum concentration is normally $42 \mu \mathrm{g} / \mathrm{mL}$, but elevated levels have been described in type 2 diabetes [6], obesity[7], and inflammatory cardiomyopathy[8].

TTR, the natural binding partner of RBP4, is a homotetrametic protein predominantly expressed by the liver and present in plasma at physiologic concentrations of $200-400 \mu \mathrm{g} / \mathrm{mL}[9,10]$. More than 100 point mutations in the TTR gene are associated with inherited forms of amyloidosis[11] known as mutant transthyretin-associated (ATTRm) amyloidosis[12]. While there is some variability in disease phenotype, the majority of ATTRm patients feature amyloidotic cardiomyopathy. Interestingly, wild-type TTR can also be amyloidogenic, forming amyloid fibrils that mainly infiltrate the myocardium[13]. The accumulation of variant and/or wild-type TTR cardiac amyloid deposits causes progressive worsening of organ function and leads to congestive heart failure[14-16].

It is widely held that ATTR amyloid diseases share a common pathobiology. Amyloid fibril formation is thought to result from a series of misfolding and aggregation steps, collectively described as the amyloid cascade[17]. The mechanism is initiated by TTR tetramer dissociation, believed to be the rate-limiting step, and followed by aggregation of non-native monomeric forms of the protein. A specific cause for destabilization and disassembly of TTR native quaternary structure is unknown; nonetheless, in vitro studies have shown that tetramer-released monomers can rapidly adopt misfolded conformations that self-associate, forming aggregates, and eventually insoluble amyloid fibrils[18].

Tissue deposited amyloid fibrils, the hallmark of all amyloid diseases, are highly organized, non-branching structures that bind Congo red dye. In TTR-associated amyloidosis, the heart is commonly a site for unremitting accumulation of extracellular amyloid fibril deposits. Diagnosis of ATTR amyloidosis frequently relies on biopsy proof of amyloid deposits, evidence of a pathologic TTR mutation in ATTRm disease, and more recently, the use of imaging techniques such as $(99 \mathrm{~m}) \mathrm{Tc}$-pyrophosphate scintigraphy $[19,20]$. Importantly, ATTR

Correspondence to: Lawreen $\mathrm{H}$. Connors, $\mathrm{PhD}$, Amyloidosis Center, Boston University School of Medicine, K-507, 715 Albany Street, Boston, MA 02118, USA. Phone: 617 638-4313; Fax: 617 638-4493; E-mail: lconnors@bu.edu

Key words: ATTRwt, ATTRm, RBP4, cardiomyopathy, cardiac amyloidosis, amyloid

Received: July 15, 2017; Accepted: August 09, 2017; Published: August 12, 2017 
Koch CM (2017) Retinol-binding protein 4 in transthyretin-associated forms of cardiac amyloidosis: differences in the pathobiologies of mutant (ATTRm) and wild-type (ATTRwt) diseases

disease-specific indicators of onset and progression are lacking, and currently available treatment options are limited, especially for those patients with ATTRwt cardiac amyloidosis.

In the present study, we examined tissue and circulating forms of RBP4 in ATTRm and ATTRwt amyloidosis. Our interest was prompted by in vitro studies demonstrating the stabilizing effect of RBP4 on native TTR $[21,23]$ and the limited information about RBP4 in ATTR cardiac diseases, despite extensive studies of the protein in other pathologies[6,24-26] particularly cardiomyopathy [8,27,28]. This report documents our 1) RBP4 histological and serological findings in ATTRm and ATTRwt; 2) assessment of baseline serological, clinical, and echocardiographic data of patients in each group; and 3) analysis of RBP4 as an indicator of ATTR amyloid disease type.

\section{Materials and methods}

\section{Study Cohorts}

Patient sera, tissue samples, and clinical data were obtained from the Boston University (BU) Amyloidosis Center repository with the approval of the BU Medical Center Institutional Review Board. Amyloid study samples were from 3 groups of patients presenting with amyloidotic cardiomyopathy: ATTRm, ATTRwt, and immunoglobulin light chain $(\mathrm{AL})$ amyloidosis. Serum from patients seen at the $\mathrm{BU}$ Amyloidosis Center for a baseline evaluation between January 2009 and June 2013 were studied. A separate group of non-amyloid controls with cardiomyopathy were obtained from the BU Cardiovascular Center. Amyloidosis was diagnosed by Congo red staining of a tissue biopsy and identification of the amyloidogenic protein was accomplished by immunohistochemistry, immunogold electron microscopy, or mass spectrometry. ATTRm amyloidosis was established by the presence of a TTR variant protein on isoelectric focusing (IEF) and a pathologic gene mutation by DNA sequencing. For ATTRwt amyloidosis, TTR was identified in the amyloid deposit in the absence of a mutant TTR gene or variant protein sequence. Tissues from patients with immunoglobulin light chain (AL) amyloidosis were used as non-ATTR cases; AL amyloidosis was diagnosed by appropriate tissue amyloid typing, histopathologic identification of clonal plasma cells in the bone marrow, detection of a monoclonal immunoglobulin light chain (LC) by serum or urine immunofixation electrophoresis, and serum free LC testing. Clinical data was obtained from the electronic health record and included details of history, physical examination, and routine laboratory studies. Study design controlled for gender, age, and comorbidities; patient study groups included males, 60 years of age or older, with triglyceride levels $<200 \mathrm{mg} / \mathrm{dL}$. Triglyceride levels were used to exclude confounders associated with diabetes; a triglyceride cut-off value of $200 \mathrm{mg} / \mathrm{dL}$ was chosen based on previous reports showing a correlation of increased RBP4 levels to obesity [7]. Control sera from healthy individuals, age- and gender-matched to our patient groups, were obtained from BioReclamation (Westbury, NY).

A patient was considered to have amyloid cardiomyopathy when presenting with brain natriuretic protein $(\mathrm{BNP})>100 \mathrm{pg} / \mathrm{mL}$ and an abnormal echocardiogram featuring interventricular septal thickening $>12 \mathrm{~mm}$, in the absence of hypertensive or valvular heart disease. Other data evaluated included cardiac troponin (cTn-I), triglycerides, uric acid, and left ventricular ejection fraction (LVEF); these measures have previously been reported to be associated with limited survival in ATTRwt amyloidosis [29,30].

\section{Congo red staining and immunohistochemical analysis}

Serial sections of formalin-fixed, paraffin-embedded tissue samples were cut to $8 \mu \mathrm{m}$ or $5 \mu \mathrm{m}$ thickness and processed for Congo red staining or immunohistochemistry, respectively. For Congo red histology, sections were deparaffinized with xylene, counterstained with Mayer's hematoxylin, stained in alkaline Congo red, and viewed by bright field and polarized light microscopy.

Indirect immunohistochemical techniques were used to detect RBP4 and TTR in human tissue sections. Primary antibodies included monoclonal mouse anti-human RBP4 (Abcam, Cambridge, MA) and polyclonal rabbit anti-human TTR antisera (Dako, Carpinteria, CA); the secondary antibody system was HRP-labelled poymer conjugated to goat anti-mouse and anti-rabbit immunoglobulins (EnVision ${ }^{\mathrm{TM}}$ + Dual Link System-HRP, Dako, Carpinteria, CA). Tissue sections were deparaffinized in xylene, dehydrated and blocked in hydrogen peroxide for $10 \mathrm{~min}$. To unmask antigen for improved recognition by the primary antibodies, slides were heated at $95 \mathrm{C}$ for 8 minutes in Citra Plus for RBP4 or AR-10 for TTR (BioGenex, San Ramon, CA). Following antigen retrieval, slides were washed, blocked (PowerBlock, BioGenex), and stained in an automated Dako Autostainer Plus system (Dako Cytomation). Using the provided Dako diluent buffer, 1:1000 antibody dilutions were performed for TTR or 1:100 for RBP4, and slides were incubated in $200 \mu \mathrm{L}$ of primary and $200 \mu \mathrm{L}$ of secondary antibody solutions. Tissue sections were counterstained with Harris' modified hematoxylin. Images were captured using bright field light microscopy, and a SPOT Insight camera and software (Diagnostic Instruments, Sterling Heights, MI).

\section{Serum retinol-binding protein 4 measurement by sandwich enzyme-linked immunosorbent assay}

To measure circulating levels of RBP4 in serum samples, a sandwich enzyme-linked immunosorbent assay (ELISA) was developed in our laboratory. Mouse monoclonal anti-human RBP4 and biotinylated polyclonal mouse anti-human RBP4 antibodies (R\&D Systems, Minneapolis, MN) were used as the capture and detection antibodies, respectively. High-binding, flat bottom 96-well microplates (R\&D Systems, Minneapolis, MN) were coated with $50 \mu \mathrm{L}$ of $1 \mathrm{ng} / \mathrm{mL}$ mouse monoclonal anti-human RBP4 antibody and stored overnight at $4{ }^{\circ} \mathrm{C}$. Plate wells were blocked with $1 \%$ BSA and washed extensively with PBS containing $0.1 \%$ tween-20. RBP4 protein $(\mathrm{R} \& \mathrm{D})$ standard solutions, ranging from $0-10 \mathrm{ng} / \mathrm{mL}$, and serum dilutions of 1:100,000 were prepared in $0.2 \%$ BSA, $0.025 \%$ tween. Standard, sample, or control solutions were added to microplate wells in triplicate. Solutions were shaken $(500 \mathrm{rpm})$ for 2 hours at room temperature. Plates were washed and biotinylated polyclonal mouse anti-human RBP4 antibody (100 ng/mL) was applied. After 2 hours, streptavidin-HRP was added and the reaction proceeded for 30 minutes at room temperature with shaking $(500 \mathrm{rpm})$. Plates were developed using $100 \mu \mathrm{L}$ of TMB and the reaction was halted with $100 \mathrm{uL}$ of $1.5 \mathrm{~N} \mathrm{H}_{2} \mathrm{SO}_{4}$. Chromogenic changes were detected with a Biotek ELx800 and data was analyzed by Gen5 software using the SPL curve fit. The intra-assay variability was less than $5 \%$ and the inter-assay variability was less than $15 \%$.

\section{Statistical analysis}

Data from ELISA measurements of serum RBP4 were analyzed using Prism 5 software (Graphpad Software, La Jolla, CA). Gaussian distribution was assessed by running D'Agostino and Pearson omnibus normality testing. Continuous variables are described as mean \pm standard error of the mean (SEM). Baseline demographic, serologic, and echocardiographic data were contrasted among the ATTRm, ATTRwt, and AL disease types using one-way ANOVA with multiple comparisons testing or Kruskal-Wallis tests. For baseline evaluation data in ATTRm and ATTRwt, associations of RBP4 with age, body 
Koch CM (2017) Retinol-binding protein 4 in transthyretin-associated forms of cardiac amyloidosis: differences in the pathobiologies of mutant (ATTRm) and wild-type (ATTRwt) diseases

mass index (BMI), triglycerides, BNP, c-TnI, interventricular septal thickness (IVST), LVEF, uric acid, or TTR were analyzed by Pearson correlation coefficients. All analyses were conducted with the use of SAS software, version 9.3 (SAS Institute). A p-value $<0.05$ was used to determine statistical significance. No adjustments for multiplicity of testing were made.

\section{Results}

\section{Serum retinol-binding protein 4 is present in ATTR cardiac tissue}

Cardiac tissues from 4 separate cases of ATTRm amyloidosis were studied; these included ATTR-L58H ( $\mathrm{n}=2)$, ATTR-V30M $(\mathrm{n}=1)$, and ATTR-V122I $(n=1)$. The presence of amyloid deposits in all cardiac tissue specimens was confirmed by Congo red staining. Representative results from a patient who was diagnosed with ATTR-L58H amyloidosis, an ATTRm disease featuring cardiac presentation[31], are shown in Figure 1. In the Congo red treated section, stain-avid areas were observed by standard light microscopy (Figure 1a); a polarized view of the same field (Figure $1 \mathrm{~b}$ ) exhibited the characteristic 'apple green' birefringence, confirming the presence of amyloid. Immunohistochemical treatment of a serial section with polyclonal anti-human TTR antibody (Figure 1c) demonstrated strong reactivity in the area that stained with Congo red, providing evidence that the amyloid deposits were constituted from TTR protein. Testing for RBP4 showed strong cytoplasmic staining in cardiomyocytes adjacent to the amyloid deposits (Figure 1d).

RBP4 histological and immunohistochemical features in ATTRwt cardiac tissue samples $(n=3)$ were also characterized. Congo red treatment provided evidence of extensive amyloid deposition in the myocardium and myocardial vasculature under standard (Figure 1e) and polarized light (Figure 1f). Immunohistochemical analyses of serial sections showed strong reactivity for TTR confirming the biochemical nature of the amyloid deposits (Figure 1g). Staining for RBP4 demonstrated the presence of RBP4 protein in the cardiomyocytes surrounding amyloid infiltrated vasculature (Figure 1h); however, the RBP4 staining in ATTRwt tissues was weaker and less uniform compared to the immunoreactivity observed in ATTRm heart samples (Supplemental Figure 1).

For comparison, autopsied heart tissue sections from a patient with cardiomyopathy in the absence of amyloid were analyzed. Congo red treatment of the tissue was negative when viewed by standard and polarized light microscopy, confirming that no amyloid fibrillar deposits were present (Supplemental Figure 2a, b). Moreover, there was no evidence of RBP4 in the tissue by immunohistochemical staining (Supplemental Figure 2c) indicating that the protein was absent in cardiac tissue lacking amyloid infiltration. Liver tissue sections from an AL (non-ATTR) amyloidosis case served as positive staining controls (Supplemental Figure 2d) since RBP4 is abundantly synthesized by hepatocytes; as expected, strong RBP4 staining was observed in the liver tissue and confirmed antibody specificity.

\section{Serum concentrations of retinol-binding protein 4 in ATTR amyloidosis}

To investigate the levels of RBP4 in amyloid patient sera and controls, we developed an ELISA method for reproducible quantification. We assessed serum levels of RBP4 in males over 60 years of age from the following groups: 1) healthy controls, 2) ATTRm amyloidosis featuring cardiomyopathy (CMP), 3) ATTRwt amyloidosis, 4) AL (non-ATTR) amyloidosis with CMP, and 5) non-amyloid with CMP.
The ATTRm group consisted of patients heterozygous for the following TTR mutations: T60A $(\mathrm{n}=11)$, V122I $(\mathrm{n}=8)$, and individual cases of D18G, V20I, G47E, I68L and S77Y. For our comparative analyses, we also chose to investigate RBP4 serum concentrations in patients with cardiomyopathy unrelated to ATTR amyloidosis, i.e. AL cardiac amyloidosis and non-amyloidotic cardiomyopathy. Control and patient serum RBP4 data are detailed in Figure 2. RBP4 was significantly decreased in ATTRm compared to healthy controls (29.11 vs. 41.83 $\mu \mathrm{g} / \mathrm{mL}, \mathrm{p}=0.0011)$ and all patient groups, including ATTRwt (29.11 vs. $42.83 \mu \mathrm{g} / \mathrm{mL}, \mathrm{p}<0.0001)$. Conversely, RBP4 levels in patients with ATTRwt were comparable to controls and not significantly different from the AL (non-ATTR) or non-amyloid CMP groups.
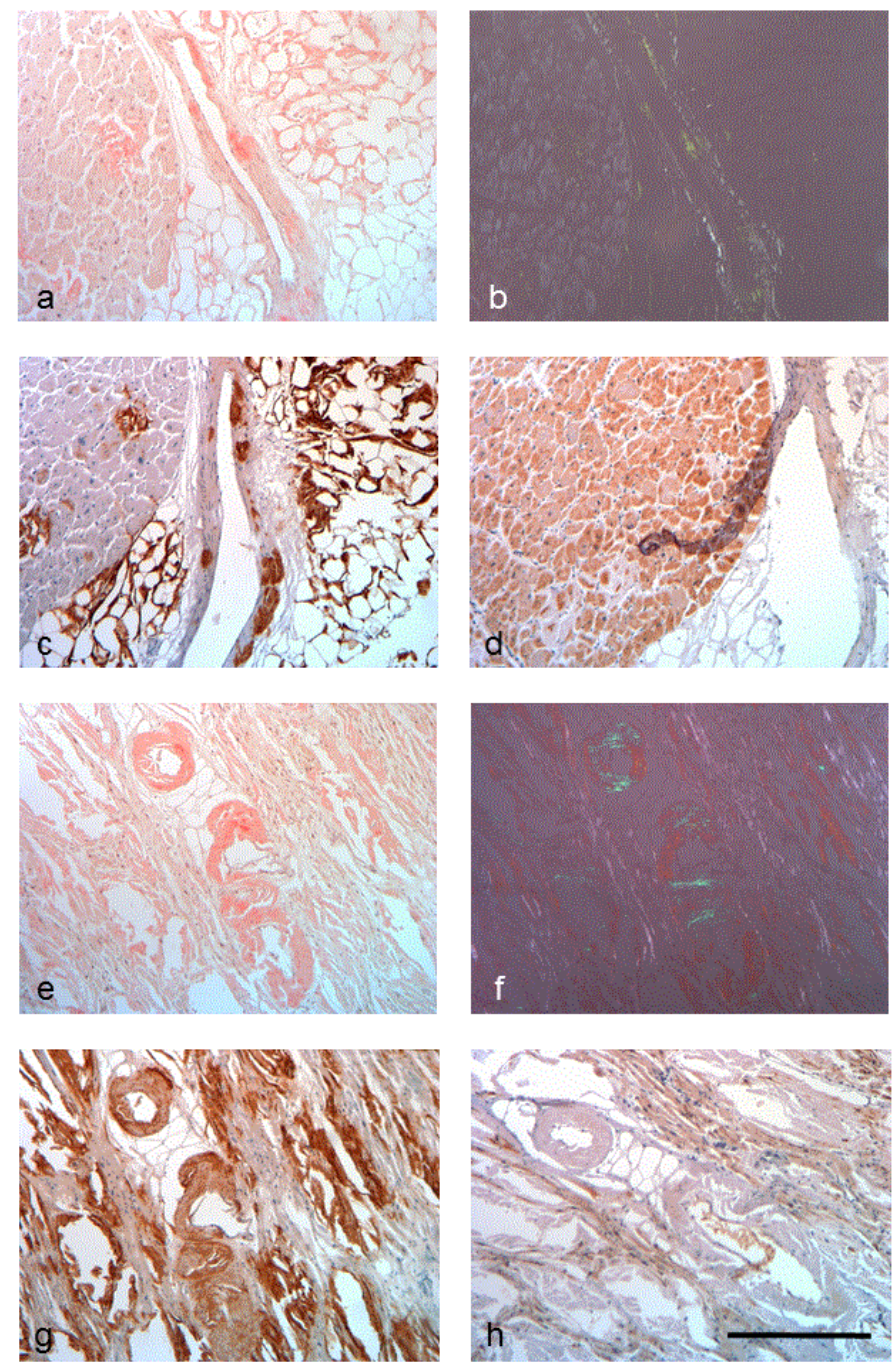

Figure 1. Histological and immunohistochemical analyses of ATTRm and ATTRw amyloidotic cardiac tissue. Serial sections of autopsied cardiac tissue from a patient with ATTRm-L58H (a-d) were stained with Congo red and viewed by (a) standard and (b) polarized light microscopy. Amyloid was demonstrated by the appearance of (a) salmon-colored deposits that exhibited (b) green birefringence on polarization Immunohistochemical staining with (c) anti-human TTR antibody confirmed the presence of TTR in the amyloid deposits and (d) anti-human RBP4 antibody staining was positive in the region adjacent to the amyloid deposition. Serial sections of autopsied cardiac tissue from a patient with ATTRwt amyloidosis (e-h) were stained with Congo red and exhibited strong areas of amyloid deposition by (e) standard light and (f) green birefringence on polarization. (g) Immunohistochemical analysis for TTR indicated the presence of the protein in the amyloid infiltrated blood vessels as well as areas proximal to the vasculature (h) anti-human RBP4 antibody staining was negative in the amyloid deposits but slightly positive in the cardiomyocytes surrounding the amyloid deposits. Original magnification $100 \mathrm{x}$. Scale bar $=100$ microns. 


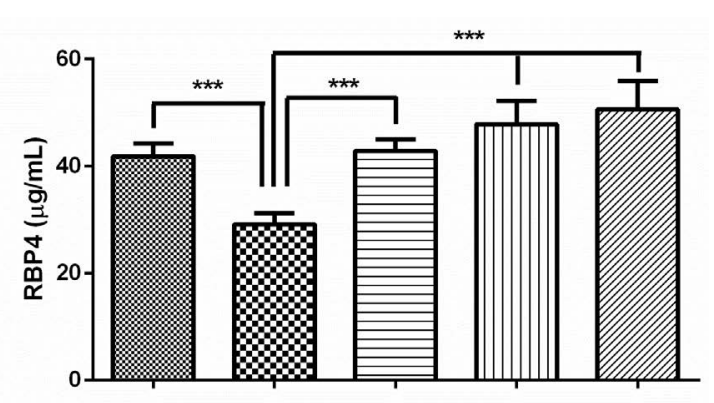

\begin{tabular}{|c|c|c|c|c|c|}
\hline Amyloid & - & ATTRm & ATTRwt & AL & - \\
\hline CMP & - & + & + & + & + \\
\hline $\mathbf{n}$ & 35 & 25 & 34 & 27 & 25 \\
\hline Average age & 64.8 & 64.6 & 75.5 & 66 & 69 \\
\hline Average RBP4 (ug/mL) & 41.83 & 29.11 & 42.83 & 47.84 & 50.58 \\
\hline
\end{tabular}

Figure 2. Levels of retinol-binding protein in control and amyloid patient sera measured by ELISA. RBP4 levels (ug/mL) were measured in serum samples from healthy controls, ATTRm (mutant TTR-associated) amyloidosis featuring cardiomyopathy, ATTRwt (wild-type TTR-associated) amyloidosis, AL (immunoglobulin light chain-associated) amyloidosis with cardiomyopathy, and non-amyloid with cardiomyopathy (CMP no amyloid). Compared to the control group, RBP4 was significantly lower in ATTRm (p $=0.0011$. In addition, RBP4 levels were significantly different between ATTRwt and $\operatorname{ATTRm}(\mathrm{p}<0.0001)$, ATTRm and AL $(\mathrm{p}=0.0016)$, and ATTRm and CMP no amyloid (p $=0.0017)$. Sera were measured in triplicate; $n$ represents the number of sera tested in each group. $* * * \mathrm{p}<0.001$.

Baseline demographic, serological and echocardiographic data in the cardiac amyloid patient groups (ATTRm, ATTRwt, and AL) are presented as mean values in Table 1A; analysis of variation among the groups is reflected in the p-values. Serum RBP4 levels (highlighted in gray) were significantly different in the group comparison with the lowest levels observed in ATTRm $(29.11 \mathrm{ug} / \mathrm{mL})$; ATTRwt concentrations were slightly lower than $\mathrm{AL}(42.83 v s .47 .84 \mathrm{ug} / \mathrm{mL})$. Serum TTR levels were significantly different $(p=0.0020)$ in ATTRm, ATTRwt, and AL; consistent with the RBP4 data, the lowest levels of TTR were found in the ATTRm group $(0.18 \mathrm{mg} / \mathrm{mL})$. While elevated levels of cardiac biomarkers were present in ATTR and AL, there was significant variation in BNP $(\mathrm{p}<0.0001)$ and cTn-I values $(\mathrm{p}<0.0244)$ among the groups. Although the mean age was higher in the ATTRwt patient cohort, other measures including BMI, triglycerides, uric acid, IVST and LVEF were comparable among the cardiac amyloid groups.

To assess relationships between baseline RBP4 serum concentrations and other previously reported measures associated with survival in ATTR [30], correlation coefficients were calculated and adjusted for age and triglyceride-status prior to analysis. RBP4 and TTR were correlated in both ATTR amyloid diseases (Table 1B) as expected. More interestingly, a strong association between RBP4 and c-TnI was identified solely in the ATTRwt cohort (Pearson $r=0.3716$, two-tailed $\mathrm{p}$-value $=0.035$ ). No other significant correlations with RBP4 were noted.

\section{Discussion}

This report documents the results of our histological and serological analyses of RBP4 in TTR-associated forms of cardiac amyloidosis. We provide evidence that RBP4 is present in cardiac tissues from patients with ATTRm and ATTRwt amyloidosis, although not associated with TTR in the amyloid fibril deposits. Our study of serum RBP4 shows that significantly lower levels are present in patients with ATTRm compared to ATTRwt $(\mathrm{p}<0.0001)$ and healthy controls $(\mathrm{p}<0.0011)$. Moreover, in a correlative analysis, we find that there is significant correlation between circulating RBP4 concentration and cardiac troponin-I (c-TnI) in patients with ATTRwt amyloidosis at baseline evaluation.
Previous in vitro studies[21,22] have provided evidence that RBP4 can extend the aggregation kinetics of thermally-denatured TTR, implying that RBP4 stabilizes the native conformation of TTR. Based on these findings, we theorized that TTR found in amyloid deposits would no longer be bound to its natural and stabilizing binding partner, RBP4. To investigate this hypothesis, we analyzed autopsied ATTR cardiac samples, heavily infiltrated with TTR amyloid, for the presence of RBP4. Congo red treatment of ATTRm and ATTRwt heart sections confirmed the presence of amyloid deposits in all tissues and immunohistochemical testing conclusively identified TTR as the amyloid fibril protein. Using antibodies specific for RBP4, the secreted form of the protein, immunoreactivity was observed in intracellular regions adjacent to amyloid deposits in ATTRm and ATTRwt cardiac tissue sections. The intracellular RBP4 appeared to be specifically concentrated in regions of the tissue surrounding amyloid laden blood vessels (Figure 1); there was no evidence of RBP4 in the extracellular amyloid deposits. In our study, the presence of RBP4 in cardiac tissue was a finding unique to the ATTR samples, as testing of heart specimens from non-amyloid cases of cardiomyopathy showed no evidence of the protein.

While RPB4 was observed in all ATTR cardiac sections, the staining profiles of ATTRm and ATTRwt samples were dissimilar. In the ATTRm samples, immunostaining was more abundant and of greater intensity compared to less pronounced results observed in the ATTRwt specimens. These differences may reflect weaker interactions between RBP4 and variant forms of TTR, i.e. decreased binding affinities specifically between RBP4 and L58H, V30M, or V122I. It has previously been reported that interactions in contact areas between RBP4 and TTR are highly sensitive to even subtle conformational changes in either protein $[32,33]$. Thus, it is possible that TTR mutant proteins in general, or those specifically associated with ATTR cardiomyopathy, feature structural configurations that decrease the binding affinity of the protein to RBP4 through steric hindrance, electrostatic or hydrophobic interference, or other factors that limit interactions. In this scenario, TTR free of RBP4 in the circulation of patients with ATTRm amyloidosis would be available and could deposit in heart tissue more readily.

In the serological analysis (Figure 2), data from ELISA measurement of serum RBP4 showed significant differences between ATTRm and ATTRwt cases featuring cardiomyopathy $(29.11$ vs. $42.83 \mathrm{~g} / \mathrm{mL}, \mathrm{p}<$ 0.0001). In fact, circulating RBP4 levels were uniquely decreased in ATTRm compared to age- and gender-matched groups that included healthy controls, and cases of AL cardiac amyloidosis or non-amyloid cardiomyopathy. These data are consistent with a recently reported study by Arvanitis et al. showing that RBP4 levels in ATTR V122I amyloidosis were significantly lower compared with non-amyloid controls[34]. In ATTRm amyloidosis, it seems reasonable that lowered RBP4 levels correlate to higher amounts of unbound (RBP4-free) TTR; thus, the energy barrier for disassociation of tetrameric TTR would be lower and amyloid fibril formation more likely to occur. Our immunohistochemical analysis demonstrating the absence of RBP4 in ATTRm cardiac amyloid deposits (Figure 1a-d) lends support to this supposition. It is important to note that serum concentrations of TTR were also lowest in the ATTRm group; whether this is due to increased amyloid deposition or an alternative cause is unclear. Conversely, RBP4 levels in the ATTRwt group were an unexpected finding. Concentrations were comparative to the healthy controls and not significantly different from the other patient groups (AL cardiac and non-amyloid cardiomyopathy). Wild-type TTR, unlike the mutant forms, contains a 'normal' amino acid sequence and higher 
Koch CM (2017) Retinol-binding protein 4 in transthyretin-associated forms of cardiac amyloidosis: differences in the pathobiologies of mutant (ATTRm) and wild-type (ATTRwt) diseases

Table 1A. Baseline demographic, serological, and echocardiographic data in cardiac amyloidosis

\begin{tabular}{|c|c|c|c|c|}
\hline & ATTRm & ATTRwt & $\mathrm{AL}$ & $\mathrm{p}$-value \\
\hline$N$ & 25 & 34 & 27 & \\
\hline \multicolumn{5}{|l|}{ Characteristic } \\
\hline Gender & male & male & male & \\
\hline Age, y & $64.6 \pm 1.74$ & $75.5 \pm 1.09$ & $66.0 \pm 0.97$ & $<0.0001 * * *$ \\
\hline BMI, $\mathrm{kg} / \mathrm{m}^{2}$ & $28.13 \pm 0.73$ & $28.79 \pm 0.64$ & $27.67 \pm 0.91$ & 0.5259 \\
\hline \multicolumn{5}{|l|}{ Serological Marker } \\
\hline $\mathrm{RBP} 4, \mu \mathrm{g} / \mathrm{mL}$ & $29.11 \pm 2.05$ & $42.83 \pm 2.13$ & $47.84 \pm 4.30$ & $0.0001 * * *$ \\
\hline Triglycerides, mg/dL & $86.25 \pm 4.98$ & $96.24 \pm 5.91$ & $106.12 \pm 7.19$ & 0.1060 \\
\hline Uric acid, $\mathrm{mg} / \mathrm{dL}$ & $7.19 \pm 0.05$ & $7.91 \pm 0.41$ & $7.73 \pm 0.38$ & 0.3619 \\
\hline $\mathrm{BNP}, \mathrm{pg} / \mathrm{mL}$ & $486.32 \pm 103.03$ & $485.15 \pm 55.12$ & $1253.0 \pm 210.23$ & $<0.0001^{* * *}$ \\
\hline $\mathrm{c}-\mathrm{TnI}, \mathrm{ng} / \mathrm{mL}$ & $0.133 \pm 0.02$ & $0.167 \pm 0.04$ & $0.551 \pm 0.32$ & $0.0244 *$ \\
\hline $\mathrm{TTR}, \mathrm{mg} / \mathrm{mL}$ & $0.18 \pm 0.009$ & $0.23 \pm 0.01$ & $0.22 \pm 0.01$ & $0.0020 * *$ \\
\hline \multicolumn{5}{|l|}{ Echocardiography } \\
\hline IVST, mm & $15.72 \pm 0.73$ & $16.88 \pm 0.50$ & $15.59 \pm 0.70$ & 0.1119 \\
\hline LVEF, \% & $44.08 \pm 2.75$ & $46.18 \pm 1.94$ & $45.56 \pm 1.75$ & 0.7829 \\
\hline
\end{tabular}

Data are presented as mean \pm standard error of the mean (SEM); ATTRm, mutant TTR-associated cardiac amyloid disease; ATTRwt, wild-type TTR-associated cardiac amyloid disease; $\mathrm{AL}$, immunoglobulin light chain-associated cardiac amyloid disease

${ }^{*} \mathrm{p}<0.05, * * \mathrm{p}<0.01, * * * \mathrm{p}<0.001$

RBP4 results are highlighted in gray

Table 1B. Associations between baseline serum RBP4 concentrations and predictors of reduced survival in ATTR amyloidosis

\begin{tabular}{|c|c|c|c|c|}
\hline & \multicolumn{2}{|c|}{ ATTRm } & \multicolumn{2}{c|}{ ATTRwt } \\
\hline Age, y & 0.17 & $(0.42)$ & 0.27 & $(0.12)$ \\
\hline BMI, kg/m & 0.14 & $(0.51)$ & -0.22 & $(0.22)$ \\
\hline Triglycerides, mg/dL & 0.13 & $(0.57)$ & 0.09 & $(0.59)$ \\
\hline Uric acid, mg/dL & -0.002 & $(0.99)$ & 0.11 & $(0.54)$ \\
\hline BNP, pg/mL & 0.13 & $(0.57)$ & 0.01 & $(0.97)$ \\
\hline c-TnI, ng/mL & -0.05 & $(0.83)$ & 0.37 & $(0.035)^{*}$ \\
\hline TTR, mg/mL & 0.53 & $(0.01)^{*}$ & 0.48 & $(0.004)^{* *}$ \\
\hline IVST, mm & 0.29 & $(0.21)$ & -0.12 & $(0.51)$ \\
\hline LVEF, \% & 0.26 & $(0.25)$ & 0.10 & $(0.57)$ \\
\hline
\end{tabular}

Pearson correlation coefficients (p-values) for each analysis are shown; ATTRm, mutan TTR-associated cardiac amyloid disease; ATTRwt, wild-type TTR-associated cardiac amyloid disease

${ }^{*} \mathrm{p}<0.05, * * \mathrm{p}<0.01$

order structure that favors interactions with RBP4; hence, elimination of RBP4, a relatively small protein, through renal filtration should be prevented and result in higher circulating levels of RBP4. A stronger molecular association between RBP4 and wild-type TTR, compared to mutant forms of the protein, would be consistent with our observation of lower amounts of RBP4 in ATTRwt cardiac tissue specimens. While the analysis of serum RBP4 concentrations does not provide direct information about RBP4-mediated TTR stabilization, these data are in line with previous reports[21,35] and our in vitro findings (data not shown) that RBP4 stabilizes TTR and slows the aggregation kinetics of the amyloid genic protein.

Our immune histochemical and serological results suggest that the role of RBP4 in the mechanism of TTR amyloid genesis differs in ATTRm and ATTRwt cardiac amyloid diseases. In ATTRm amyloidosis, weakened TTR-RBP4 associations may result in lower levels of serum RBP4 and a higher degree of TTR amyloid deposition in cardiac tissue. In contrast, a tighter interaction between wild-type TTR and RBP4 would explain higher amounts of the circulating complexes in ATTRwt amyloidosis, but suggests that factors other than TTR-RBP4 pairing must contribute to amyloid fibril formation in this disease. It seems plausible that the cardiac tissue environment may contain key features, yet to be defined, that contribute to the pathobiological dissimilarities we have observed in ATTRm vs. ATTRwt. In addition to differences in TTR-RBP4 pairing associations, variations in receptor-mediated targeting and/or cell signaling pathways may be important, especially in ATTRwt amyloidosis. Studies published over the last thirty years have demonstrated the existence of several RBP4-specific receptors in a variety of tissues including the choroid plexus, testes, kidney, and eye. One such receptor, STRA6, allows RBP4 to transfer vitamin A across cell membranes for binding to intracellular isoforms of RBP (CRBP) [36-40]. A similar receptor, with specificity for TTR-RBP4 complexes, may be present in cardiac tissue. In a cascade of events, conformational changes in RBP4 resulting from interactions with a cardiac cell surface receptor would lead to weakening of the TTR-RBP4 interactions, destabilization of tetrameric TTR, subunit dissociation, and initiation of the amyloid fibril formation pathway.

The present study was limited by small sample sizes in both the histological and serological analyses. Several constraining factors included the study selection criteria which were age $>60$ years, male sex, and exclusion of all patients participating in ATTR clinical trials or taking investigational drugs at the time of blood draw. In addition, while sera are routinely collected at all baseline evaluations and readily available from our repository, cardiac tissues were restricted to a small set of autopsy specimens. Expansion of these studies to larger group numbers would confirm our findings and further demonstrate the utility of RBP4 as a biomarker of ATTRm cardiac amyloid disease. Moreover, this study did not include examination of serum vitamin A levels, potentially important as retinol is a key player in the recognition and binding of RBP4 to TTR [4, 21, 41]. Unfortunately, accurate measurement of vitamin A is challenging as levels are homeostatically controlled (50-200 g/dL) [42] and serum retinol concentrations are only an indirect reflection of total vitamin $\mathrm{A}$ in the liver. Obtaining these data in future studies may be key as normal homeostasis of vitamin A is interrupted in several conditions where decreased levels $(<0.7 \mu \mathrm{g} / \mathrm{dL})$ have been reported[43-46] and retinoid insufficiency has been linked to alterations in the extracellular matrix of the heart which can affect adult cardiac function [47].

\section{Conclusion}

In summary, results of our study of cardiac tissues and sera from patients with ATTRm or ATTRwt amyloidosis suggest that the pathobiologies of the two diseases are dissimilar with respect to RBP4. We demonstrate, for the first time, that RBP4 is uniquely present in 
Koch CM (2017) Retinol-binding protein 4 in transthyretin-associated forms of cardiac amyloidosis: differences in the pathobiologies of mutant (ATTRm) and wild-type (ATTRwt) diseases

cardiac tissues from patients with TTR-associated cardiac amyloid. While not co-deposited with TTR fibrils, RBP4 appeared to be present in locations adjacent to the cardiac amyloid deposits more abundantly in ATTRm tissues compared to ATTRwt specimens. Moreover, circulating levels of RBP4 were significantly lower in ATTRm compared to ATTRwt $(\mathrm{p}<0.0001)$ and healthy controls $(\mathrm{p}<0.0011)$. Lastly, we found a correlation between circulating RBP4 levels and cardiac troponin-I (c-TnI) in ATTRwt, as well as expected strong correlations between RBP4 and TTR in both ATTRm and ATTRwt. These data suggest that RBP4 may have utility as a serum biomarker in ATTRm amyloidosis and provide evidence in support of a therapeutic strategy that takes advantage of the stabilizing effects of a natural binding partner of TTR.

\section{Acknowledgements}

This work was supported by the Young Family Amyloid Research Fund, NIH RO1AG031805 (LHC), NIH U54TR001012 (FLR), and NIH R21AG050206 (FLR). The current affiliation of Dr. Clarissa M. Koch is Department of Medicine, Division of Pulmonary \& Critical Care, Northwestern University, Chicago, IL. Dr. Evan L. Chiswick is presently affiliated with Massachusetts Institute of Technology, Boston, MA.

\section{Conflict of interest}

All authors declare no conflict of interest

\section{Reference}

1. D. Bellovino, T. Morimoto, F. Tosetti, S. Gaetani (1996) Retinol binding protein and transthyretin are secreted as a complex formed in the endoplasmic reticulum in HepG2 human hepato carcinoma cells. Exp. Cell Res 222: 77-83.

2. H. Melhus, T. Nilsson, P. a Peterson, L. Rask (1991) Retinol-binding protein and transthyretin expressed in HeLa cells form a complex in the endoplasmic reticulum in both the absence and the presence of retinol. Exp. Cell Res 197: 119-24.

3. H. Ronne, C. Ocklind, K. Wiman, L. Rask, B. Obrink, P. A. Peterson (1983) Liganddependent regulation of intracellular protein transport: effect of vitamin a on the secretion of the retinol-binding protein. J. Cell Biol 96: 907-910.

4. S. Hyung, S. Deroo, C. Robinson (2010) Retinol and retinol-binding protein stabilize transthyretin via formation of retinol transport complex. ACS Chem. Biol 5: 1137-1146.

5. M. M. Sousa, M. J. Saraiva (2001) Internalization of transthyretin. Evidence of a novel yet unidentified receptor-associated protein (RAP)-sensitive receptor. J. Biol. Chem 276: 14420-5. [Crossref]

6. Graham TE, Yang Q, Blüher M, Hammarstedt A, Ciaraldi TP, et al. (2006) Retinolbinding protein 4 and insulin resistance in lean, obese, and diabetic subjects. $N$ Engl $J$ Med 354: 2552-2563. [Crossref]

7. Peter Kovacs, Christopher J. Wason, Mathias Fasshauer, Michael R. Schön, N. Klöting et al. (2007) Serum retinol-binding protein is more highly expressed in visceral than in subcutaneous adipose tissue and is a marker of intra-abdominal fat mass. Cell Metab 6: $79-87$.

8. Bobbert P, Weithäuser A, Andres J, Bobbert T, Kühl U, et al. (2009) Increased plasma retinol binding protein 4 levels in patients with inflammatory cardiomyopathy. Eur. $J$. Heart Fail 11: 1163-1168. [Crossref]

9. R. F. Ritchie, G. E. Palomaki, L. M. Neveux, O. Navolotskaia, T. B. Ledue, et al. (1999) Reference distributions for the negative acute-phase serum proteins, albumin, transferrin and transthyretin: a practical, simple and clinically relevant approach in a large cohort. J. Clin. Lab. Anal 13: 273-279. [Crossref]

10. J. Buxbaum, I. Anan, O. Suhr (2010) Serum transthyretin levels in Swedish TTR V30M carriers. Amyloid 17: 83-85. [Crossref]

11. Connors LH, Lim A, Prokaeva T, Roskens VA, Costello CE (2003) Tabulation of human transthyretin (TTR) variants, 2003. Amyloid 10: 160-184. [Crossref]

12. K. B. Shah, Y. Inoue, M. R. Mehra (2006) Amyloidosis and the heart: a comprehensive review. Arch. Intern. Med 166: 1805-1813.

13. P. Westermark, K. Sletten, B.Johansson, G.G.Cornwell (1990) Fibril in senile systemic amyloidosis is derived from normal transthyretin. Proc. Natl. Acad. Sci. U. S. A 87: 2843-2845. [Crossref]
14. B. Ng, L. Connors, R. Davidoff, M. Skinner, R. Falk (2005) Senile systemic amyloidosis presenting with heart failure: a comparison with light chain-associated amyloidosis. Arch. Intern. Med 165: 1425-1429. [Crossref]

15. Pitkänen P, Westermark P, Cornwell GG 3rd (1984) Senile systemic amyloidosis. Am J Pathol 117: 391-399. [Crossref]

16. Ueda M, Horibata Y, Shono M, Misumi Y, Oshima T, et al. (2011) Clinicopathological features of senile systemic amyloidosis: an ante- and post-mortem study. Mod Pathol 24: 1533-1544. [Crossref]

17. Knowles TP, Vendruscolo M1, Dobson CM (2014) The amyloid state and its association with protein misfolding diseases. Nat Rev Mol Cell Biol 15: 384-396. [Crossref]

18. Quintas A, Vaz DC, Cardoso I, Saraiva MJ, Brito RM (2001) Tetramer dissociation and monomer partial unfolding precedes protofibril formation in amyloidogenic transthyretin variants. J. Biol. Chem 276: 27207-27213

19. F. Badalà, K. Nouri-mahdavi, D. A. Raoof (2008) “99mTc-Pyrophosphate scintigraphy for differentiating light-chain cardiac amyloidosis from transthyretin-related familial and senile cardiac amyloidosis. Computer (Long. Beach. Calif) 144: 724-732.

20. Dungu JN, Valencia O, Pinney JH, Gibbs SD, Rowczenio D, et al. (2014) CMR-based differentiation of AL and ATTR cardiac amyloidosis. JACC Cardiovasc Imaging 7 : 133-142. [Crossref]

21. J. T. White, J.W. Kelly (2001) Support for the multigenic hypothesis of amyloidosis: the binding stoichiometry of retinol-binding protein, vitamin A, and thyroid hormone influences transthyretin amyloidogenicity in vitro. Proc. Natl. Acad. Sci. U. S. A 98 13019-13024. [Crossref]

22. Mangione PP, Porcari R, Gillmore JD, Pucci P, Monti M, et al. (2014) Proteolytic cleavage of Ser52Pro variant transthyretin triggers its amyloid fibrillogenesis. Proc. Natl. Acad. Sci. U. S. A 111: 1539-1544. [Crossref]

23. Monaco HL (2000) The transthyretin-retinol-binding protein complex. Biochim Biophys Acta 1482: 65-72. [Crossref]

24. Anette Bachmann, Jeannette Seeger, Ulrike Lossner, M. Ziegelmeier, Mathia Fasshauer, et al. (2007) Serum levels of adipokine retinol-binding protein-4 in relation to renal function. Diabetes Care 30: 2588-2592.

25. K. Krzyzanowska, C. Schnack, R. Rahman, J. Brix, B. B. Kahn, et al. (2008) Serum concentrations of retinol-binding protein 4 in women with and without gestational diabetes. Diabetologia 51: 1115-1122. [Crossref]

26. Yang Q, Graham TE, Mody N, Preitner F, Peroni OD, et al. (2005) Serum retinol binding protein 4 contributes to insulin resistance in obesity and type 2 diabetes. Nature 436 : 356-362. [Crossref]

27. Sun Q, Kiernan UA, Shi L, Phillips DA, Kahn BB, et al. (2013) Plasma retinol-binding protein 4 (RBP4) levels and risk of coronary heart disease: a prospective analysis among women in the nurses health study. Circulation 127: 1938-1947. [Crossref]

28. J. Wang, H. Chen, Y. Liu, W. Zhou, R. Sun, et al. (2015) Retinol binding protein 4 induces mitochondrial dysfunction and vascular oxidative damage. Atherosclerosis 240: $335-344$.

29. Pinney JH, Whelan CJ, Petrie A, Dungu J, Banypersad SM, et al. (2013) Senile systemic amyloidosis: clinical features at presentation and outcome. J. Am. Heart Assoc 2: e000098. [Crossref]

30. L. H. Connors, Flora Sam, Martha Skinner, Francesco Salinaro, Fangui Sun, et al. (2015) Heart Failure Due to Age-Related Cardiac Amyloid Disease Associated With Wild-Type Transthyretin: A Prospective, Observational Cohort Study. Circulation 133: 282-290. [Crossref]

31. Ruberg FL, Berk JL (2012) Transthyretin (TTR) cardiac amyloidosis. Circulation 126 1286-1300. [Crossref]

32. G. Zanotti, R. Berni (2004) Plasma retinol-binding protein: structure and interactions with retinol, retinoids, and transthyretin. Vitam Horm 69: 271-295. [Crossref]

33. Liz MA, Faro CJ, Saraiva MJ, Sousa MM (2004) Transthyretin, a new cryptic protease. J Biol Chem 279: 21431-21438. [Crossref]

34. Arvanitis M, Koch CM, Chan GG, Torres-Arancivia C, LaValley MP, et al. (2017) Identification of Transthyretin Cardiac Amyloidosis Using Serum Retinol-Binding Protein 4 and a Clinical Prediction Model. JAMA Cardiol 2118: 305-313. [Crossref]

35. Heller J, Horwitz J (1974) The binding stoichiometry of human plasma retinol-binding protein to prealbumin. J Biol Chem 249: 5933-5938. [Crossref]

36. D.C. Berry, S.M. O’Byrne, A.C.Vreeland, W. S. Blaner, N. Noy (2012) Cross talk between signaling and vitamin A transport by the retinol-binding protein receptor STRA6. Mol. Cell. Biol 32: 3164-3175. [Crossref] 
Koch CM (2017) Retinol-binding protein 4 in transthyretin-associated forms of cardiac amyloidosis: differences in the pathobiologies of mutant (ATTRm) and wild-type (ATTRwt) diseases

37. C. Redondo, M. Vouropoulou, J. Evans, J. B. C. Findlay (2008) Identification of the retinol-binding protein (RBP) interaction site and functional state of RBPs for the membrane receptor. FASEB J 22: 1043-1054.

38. M. Sundaram, A. Sivaprasadarao, M. M. DeSousa, J. B. Findlay (1998) The transfer of retinol from serum retinol-binding protein to cellular retinol-binding protein is mediated by a membrane receptor. J. Biol. Chem 273: 3336-3342.

39. B. A. Pfeffer, V. M. Clark, J. G. Flannery, D. Bok (1986) Membrane receptors for retinol-binding protein in cultured human retinal pigment epithelium. Invest Ophthalmol Vis Sci 27: 1031-1040

40. Blaner WS (2007) STRA6, a cell-surface receptor for retinol-binding protein: the plot thickens. Cell Metab 5: 164-166. [Crossref]

41. H. M. Naylor, M. E. Newcomer (1999) The structure of human retinol-binding protein (RBP) with its carrier protein transthyretin reveals an interaction with the carboxy terminus of RBP. Biochemistry 38: 2647-2653. [Crossref]

42. Russell RM (2000) The vitamin A spectrum: from deficiency to toxicity. Am J Clin Nutr 71: 878-884. [Crossref]
43. Hans K Biesalski, Jürgen Frank, Susanne C Beck, Felix Heinrich, Beate Illek, et al. (1999) "Biochemical but not clinical vitamin A deficiency results from mutations in the gene for retinol binding protein. Am J Clin Nutr 69: 931-936.

44. V. Lopez-Teros, Justin Chileshe, Nicole Idohou-Dossou, Tetra Fajarwati, Gabrie Medoua Nama, et al. (2014) International experiences in assessing vitamin a status and applying the vitamin A-labeled isotope dilution method. Int J Vitam Nutr Res 84: $40-51$.

45. T. Konstantyner, S. Warkentin, J. A. de A. C. Taddei (2014) Prevalence and determinants of vitamin A deficiency among Brazilian children under 2 years of age from the 2006 National Demographic Health Survey. Food Nutr Bull 35: 422-430.

46. Trasino SE, Benoit YD, Gudas LJ (2015) Vitamin A deficiency causes hyperglycemia and loss of pancreatic $\hat{I}^{2}$-cell mass. J Biol Chem 290: 1456-1473. [Crossref]

47. Christopher C. Wendler, Angela Schmoldt, George R. Flentke, Lauren C. Case, Loredana Quadro et al. (2003) Increased fibronectin deposition in embryonic hearts of retinol-binding protein-null mice. Circ. Res 92: 920-928. [Crossref]

Copyright: $(2017$ Gatti AM. This is an open-access article distributed under the terms of the Creative Commons Attribution License, which permits unrestricted use, distribution, and reproduction in any medium, provided the original author and source are credited. 